\title{
GOVERNANDO O ENSINO SUPERIOR Lições da Grã-Bretanha para o Brasil
}

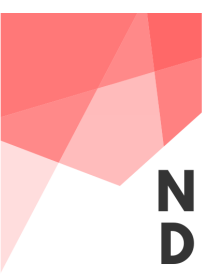

\author{
GOVERNING HIGHER EDUCATION \\ Lessons from Britain to Brazil
}

\author{
John Gledhill \\ The University of Manchester \\ School of Social Sciences | Manchester, Inglaterra \\ johngled@me.com | ORCID iD: 0000-0002-4275-2556
}

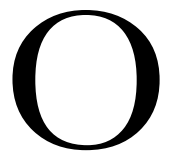

que Marilyn Strathern (1996) chamou de "cultura de auditoria" transformou a vida profissional dos acadêmicos universitários, provocando uma crescente onda de descontentamento. No entanto, como Strathern demonstra ao reunir uma coleção de ensaios que abrangia culturas de auditoria em outros tipos de organizações (Strathern 2000), este também se mostrou um campo fértil para a análise antropológica crítica. A implementação de sistemas de avaliação e auditoria no ensino superior tem se distinguido de forma considerável entre os países europeus (Sivertsen 2017), e os Estados Unidos é, novamente, um caso diferente devido à riqueza de suas universidades privadas de elite e ao papel mais limitado que tem o governo federal (Brenneis 2009). Ainda assim, a experiência do Reino Unido continua central na literatura sobre a universidade enquanto pioneira cujos sistemas têm sido objeto de contínua transformação e da qual podemos tirar ensinamentos. Meu objetivo aqui é apontar alguns ensinamentos relevantes para o Brasil em um mundo de rankings universitários e métricas de desempenho e qualidade no qual acadêmicos têm sido submetidos a um "controle"1 que possuem

\footnotetext{
${ }^{1}$ N.T.: Em inglês, "accountability". Este termo é central no debate em questão, pois marca a adoção de uma linguagem e práticas manageriais pelas universidades, remetendo à ideia de mecanismos de controle e responsabilização ética e jurídica pelos atos de indivíduos e instituições que devem prestar contas ao Estado e aos cidadãos. Sua tradução para o
} 
consequências perversas devido a seu caráter coercitivo e competitivo, tanto em nível individual quanto institucional.

Devo começar declarando a posição a partir da qual escrevo. Eu mesmo já fiz muitos tipos de avaliações, tanto no México como no Reino Unido, mas também tive oportunidades de representar minha disciplina nacionalmente nas negociações com agências governamentais sobre as regras dos processos de avaliação a serem implementados. De 1997 a 2001, atuei como coordenador da Conferência Permanente dos Chefes de Departamentos de Antropologia (do Reino Unido) e fui presidente da Associação de Antropólogos Sociais (ASA) de 2005 a 2009. É possível vencer algumas batalhas. Fomos bemsucedidos, por exemplo, em derrotar a tentativa da primeira agência do Reino Unido encarregada de auditar a qualidade do ensino universitário para tratar a antropologia como uma subdisciplina da sociologia. Certamente não ganhamos a guerra que muitos antropólogos gostariam de travar sobre a forma como as universidades são "governadas" através de processos de auditoria. Isto não é surpreendente, dado que o que aconteceu com as universidades é apenas parte de uma transformação global da administração da sociedade e da vida pública (Shore e Wright 2015). "Global" aqui inclui as universidades chinesas subindo inexoravelmente no ranking mundial. No entanto, a experiência britânica mostra que grupos organizados de acadêmicos, apoiados por fortes órgãos profissionais, sociedades instruídas e academias nacionais, podem ter algum sucesso em resistir às tentativas dos gerentes universitários e órgãos governamentais de impor modelos baseados em métricas comprovadamente insatisfatórias para avaliar o ensino e a pesquisa. As regras e procedimentos que regem nossos exercícios periódicos de avaliação da pesquisa nacional mudaram de forma importante ao longo dos anos, mas sempre após consulta à comunidade acadêmica. Dada a capacidade da comunidade de questionar nossos mestres, até hoje não experimentamos

português sendo obtusa, o termo é amplamente empregado em inglês pela literatura brasileira sobre sistemas políticos e práticas administrativas privadas e públicas. Em suma, ele evoca uma teia complexa de valores como transparência, dever de prestação de contas, justificativas para decisões tomadas ou não, bem como o controle sobre gastos e o trabalho realizado. Como se trata de um termo de significado específico a certas áreas, optamos pela tradução por "controle", em consonância com o sentido atribuído por boa parte da literatura em ciências política sobre accountability e democracia que o emprega para falar de mecanismos de controle democrático (Cf. Argelina Cheibub Figueiredo. 2001. "Instituições e Política no Controle do Executivo". Dados 44(4):689-727.). 
mudanças nas regras em vigor no início de um período de avaliação durante esse mesmo período, como aconteceu recentemente no caso da avaliação de programas de pósgraduação pela Capes no Brasil, uma avaliação na qual está em jogo a própria sobrevivência de muitos programas em vez de simplesmente seu nível de apoio financeiro.

A cultura de auditoria nas universidades britânicas foi inicialmente um produto da adesão de Margaret Thatcher a soluções neoliberais diante do mal-estar pós-imperial da desindustrialização e do declínio econômico, fortemente enraizado na ideologia pessoal de uma mulher que não nasceu no seio da elite e que adotou uma leitura liberal-meritocrática dos argumentos dos altos sacerdotes da alternativa neoliberal ao capitalismo pós-guerra de vertente social-democrata, Milton Friedman e Friedrich Hayek. Uma fervorosa defensora do "redução do Estado" e da privatização das empresas públicas, Thatcher, que se tornou Primeira Ministra em 1979, insistiu que "a sociedade" não existia, mas apenas "indivíduos" que só podiam prosperar assumindo a responsabilidade por seu próprio futuro. Uma consequência acadêmica da opinião da Primeira Ministra foi que o Social Science Research Council [Conselho de Pesquisa em Ciências Sociais] foi obrigado a mudar seu nome para Economic and Social Research Council [Conselho de Pesquisa Econômica e Social], já que a Dama de Ferro insistiu que não poderia existir uma "ciência" da "sociedade".

Na segunda metade dos anos 1980, com a austeridade neoliberal já arraigada, o governo Thatcher introduziu medidas para garantir "controle e transparência" na alocação de financiamento público às universidades para pesquisa e ensino. Shore e Wright (1999) analisaram isto como um regime neoliberal de governabilidade no sentido foucaultiano (Lemke 2001), essencialmente coercitivo por natureza, mas projetado para produzir sujeitos autorreguladores disciplinados através de tecnologias de poder em que o "governo" poderia ser realizado "à distância", como disse Nikolas Rose (1999). "Auditoria" é um termo emprestado das finanças. A existência de órgãos de auditoria independentes tem claramente sua utilidade no sentido de garantir um "bom governo". Um exemplo recente é a exigência de "transparência" do governo Boris Johnson por parte do British National Audit Office [Escritório Nacional Britânico de Auditoria] em relação aos bilhões de libras esterlinas que ele desembolsou durante a crise da Covid-19 para empreiteiros do setor privado de bens e serviços, incluindo consultorias, sem 
licitação. A incapacidade de muitos contratos em produzir resultados aceitáveis gerou denúncias de "capitalismo de compadrio", dada a existência de laços pessoais entre políticos conservadores e muitas das empresas escolhidas. Ainda assim, a reputação dos quatro conglomerados de auditoria financeira dominantes globalmente, PWC, EY, Deloitte e KPMG, não permaneceram ilibados nos anos que sucederam o escândalo Enron e o colapso financeiro de 2008. Tudo isso nos convida a questionar a ideia central do paradigma da "Nova Gestão Pública" promovido pelos governos neoliberais, de que a aplicação dos princípios da empresa privada ao setor público resultará em ganhos de eficiência e melhor "relação custobenefício" para o contribuinte. Através da aplicação de "indicadores-chave de desempenho" e sua expressão em números que fornecem a base para rankings e tabelas de classificação, a maioria dos serviços públicos, desde escolas e hospitais até assistência social para idosos, é agora avaliada em termos de medidas monetárias, casando assim "gestão científica" com "um projeto de financeirização e uma nova ética de controle" (Shore e Wright 2015:425).

Esta lógica tornou-se central em minha própria universidade no início do novo milênio, após a nomeação como seu chefe de um controverso promotor do novo pensamento neoliberal da Austrália. Até mesmo a avaliação do desempenho do pessoal individual passou a incluir uma medida financeira de "contribuição" em termos de bolsas de pesquisa, número de estudantes ensinados e supervisionados, e outras formas de renda gerada para a instituição. A governança universitária foi transformada de um colegiado em um sistema gerencial "de cima para baixo" no qual o pessoal só poderia acessar o próximo nível na hierarquia através de um "gerente de linha" designado. Oportunidades de expressar preocupações diretamente à "alta direção" eram restritas a rituais públicos de consulta pouco frequentes, com pouca margem para debate e pesquisas on-line de "satisfação" do pessoal. As pesquisas on-line também são usadas local e nacionalmente para medir a "satisfação" dos estudantes com sua "experiência da universidade", seus resultados sendo traduzidos em rankings de departamentos e instituições.

O "Governo à distância" tornava mais intensa a pressão diária sobre os acadêmicos. Eles dedicavam cada vez mais tempo à preparação para a avaliação de desempenho e sessões de "desenvolvimento pessoal", participando de programas 
obrigatórios de treinamento abordando agendas empresariais assim como tarefas profissionais essenciais, e alimentando dados em sistemas on-line registrando uma miríade de atividades quantificáveis, tal treinamento e apoio pastoral sendo oferecidos aos estudantes, definindo as metas e os "resultados de aprendizagem" esperados de seus cursos, o "progresso" de seus pós-graduandos, a "produção" resultante de suas próprias pesquisas concluídas, e seus planos para pesquisas futuras. Como a obtenção de competitivas bolsas de pesquisa com financiamento externo é um "indicador-chave de desempenho", o tempo que era preciso dedicar aos processos de inscrição que se tornavam cada vez mais complicados à medida que novos critérios, incluindo o impacto não acadêmico, foi acrescido à lista de questões a serem abordadas, e a verificação interna das propostas aumentou, embora as taxas de sucesso muitas vezes estivessem abaixo de vinte e cinco por cento. A pressão da auditoria obrigou o pessoal a trabalhar muito mais horas por semana do que aquelas para as quais são contratados, em detrimento da vida familiar. As greves contra a deterioração da remuneração e das condições de trabalho, o aumento das contribuições para a aposentadoria e a redução dos benefícios na aposentadoria são mais frequentes. No entanto, enquanto outros funcionários universitários têm sofrido frequentes congelamentos salariais, os generosos salários e acordos de pensão da alta administração universitária se tornaram um escândalo que até mesmo o atual governo conservador aceita como um problema.

A responsabilidade por esses desdobramentos (e a paralela deterioração no sistema nacional de saúde universal do Reino Unido) não pode, no entanto, ser totalmente imputados aos governos conservadores. Quando o Partido Trabalhista voltou ao poder sob Tony Blair em 1997, seu governo centrista do "Novo Trabalhismo" deu continuidade ao neoliberalismo de seus antecessores conservadores relativamente ao papel do setor privado na saúde, bem-estar social e educação. Embora a versão de Blair da socialdemocracia tenha aceitado a necessidade de intervenções governamentais para aumentar a "igualdade de oportunidades" para os jovens desfavorecidos por classe, etnia e cor, ela manteve o princípio neoliberal de que os indivíduos adultos têm o dever moral de "comercializar a si mesmos" e aceitar quaisquer oportunidades que o mercado de trabalho capitalista possa oferecer. 
O governo de Blair reintroduziu as mensalidades nas universidades públicas inglesas, em 1998, substituindo o sistema anterior de bolsas de subsistência baseadas em critérios econômicos por empréstimos para todos, exceto os estudantes mais pobres. A filosofia por trás desta mudança era que os graduados universitários na condição de indivíduos, e não o contribuinte, deveriam pagar por uma educação que aumentasse seus ganhos mais tarde na vida. As taxas foram substancialmente majoradas durante o governo da coalizão liberal-conservadora que acabou com a permanência dos trabalhistas no poder em 2010, colocando cada vez mais em questão a racionalidade do sistema de empréstimos estudantis. O ônus do pagamento de suas dívidas está cada vez mais asfixiando aqueles que de fato ganham o suficiente para pagá-la. No entanto, o governo espera atualmente que apenas um quarto dos graduados ganhe o suficiente para pagar seus empréstimos na totalidade, de modo que grande parte do ônus do financiamento do ensino superior retornará ao contribuinte. Esta contradição, no entanto, levou o atual governo conservador a falar em eliminar as formações de "baixo valor" que não garantem salários mais altos. As Humanidades são um alvo preferencial, particularmente porque a questão de seu benefício econômico para o indivíduo também pode estar ligada a causas ideológicas caras aos corações conservadores, como atacar a "teoria crítica da raça". Os baixos salários auferidos por pessoas altamente qualificadas em setores como enfermagem, assistência social ou organizações beneficentes e não governamentais que normalmente seriam considerados benéficos para a sociedade, no país e no exterior, raramente figuram nestes debates. Os leitores brasileiros reconhecerão estes argumentos, já que todos eles têm sido mobilizados sob o governo Bolsonaro. A questão da privatização das universidades públicas é também cada vez mais relevante para o Brasil. As universidades totalmente privadas são de importância insignificante no Reino Unido em comparação com o Brasil, mas apesar do fato de que o Brasil possui algumas boas universidades privadas, bem como muitas outras de baixa qualidade, as universidades públicas são os principais centros de excelência em pesquisa internacional do país e são as que mais contribuem para a promoção de mobilidade social através da ampliação do acesso ao ensino superior.

As reduções no financiamento do governo tornaram as universidades britânicas dependentes da renda das mensalidades e da renda que recebem pelo fornecimento de outros serviços aos 
estudantes, especialmente hospedagem. As taxas podem chegar a 9.250 libras esterlinas por ano para estudantes domésticos e a uma média entre 12.000 e 20.000 libras esterlinas para estudantes internacionais. As universidades públicas têm sido essencialmente "mercantilizadas", obrigadas a se vender a estudantes clientes em um mercado globalmente competitivo e a investir em sua imagem e marca. As taxas mais altas pagas pelos estudantes internacionais subsidiam tanto a pesquisa como o ensino. Isto explica porque, enquanto o Brasil persistiu com o ensino exclusivamente on-line, as universidades britânicas trouxeram os estudantes de volta ao campus para uma prometida mistura de ensino presencial e on-line em setembro de 2020, apesar de uma emergente "segunda onda" de infecções por Covid-19 na Europa e dos avisos dos cientistas sobre os efeitos já observados após a reabertura dos campi americanos. A Universidade de Manchester organizou até mesmo voos fretados para trazer estudantes de alta renda da China, que constituem um em cada oito de sua população estudantil. As consequências epidemiológicas da reabertura dos campi foram previsivelmente desastrosas, tanto para as comunidades anfitriãs quanto para os próprios estudantes. As taxas de infecção por Covid dispararam e restrições mais rigorosas foram impostas a negócios locais. Uma "experiência estudantil" insatisfatória em termos de "custobenefício" e isolamentos mal geridos nos alojamentos universitários produziram reclamações de estudantes de que a administração priorizava o dinheiro em detrimento de sua segurança e demanda por abatimento nas taxas e aluguéis. Os estudantes que protestavam receberam apoio de parte do pessoal, mas boa parte do público que comentava as reportagens de jornal não os apoiaram, reclamando sobre festas ilegais de estudantes e jovens privilegiados se formando em cursos "inúteis" em tópicos como literatura. As contradições da universidade pública "mercantilizada" são apenas uma entre as muitas reveladas por uma pandemia cujos impactos diferenciais na saúde estão claramente relacionados às desigualdades socioeconômicas e étnico-raciais. O governo Johnson pode tentar impor um retorno à formação "vocacionada" que atenda às exigências do mercado de trabalho para estudantes desfavorecidos para os quais as políticas de "ampliação do acesso" abriram portas para o tipo de educação universitária liberal anteriormente restrita a estudantes de classe média e alta. Outra advertência para o Brasil? 
Concluirei esta discussão concentrando-me na avaliação da pesquisa, uma área na qual muito do que a Grã-Bretanha foi pioneira tem sido exportado, mesmo que muitas vezes tendo se "perdido na tradução". Em 1986, o governo Thatcher instituiu uma avaliação nacional periódica da qualidade da pesquisa realizada nas universidades britânicas que atribuía uma nota a cada "unidade de avaliação", principalmente departamentos disciplinares nas iterações iniciais do exercício. A nota recebida determinava o tamanho dos pacotes de subvenção que as instituições recebiam do governo para apoiar a infraestrutura de pesquisa e os salários dos pesquisadores permanentes, a base de referência de um "sistema duplo de apoio" no qual o restante do financiamento para projetos de pesquisa provém de competitivos aportes concedidos pelos conselhos nacionais de pesquisa.

No modelo original de "Research Assessment Exercise" [Aplicação de Avaliação de Pesquisa] (RAE), a avaliação era baseada na revisão por pares por comitês temáticos especializados. O princípio básico que surgiu foi que a qualidade era mais importante do que a quantidade: até quatro publicações por acadêmico em cada unidade de avaliação seriam lidas e classificadas. Foram consideradas as circunstâncias que poderiam reduzir a "produtividade" individual durante um período de revisão (como o nascimento de um filho). No entanto, surgiram problemas ao permitir que as unidades de avaliação excluíssem totalmente de sua submissão aqueles membros considerados como não sendo "ativos na pesquisa", além do óbvio de equiparar a pontuação geral das unidades com a submissão parcial e completa de membros. Os gerentes podiam ameaçar indivíduos com a mudança de seus contratos para modalidades de "dedicação exclusiva ao ensino". Indivíduos podem ser excluídos porque não se esperava que suas publicações teriam uma boa pontuação. Todo o processo da RAE promoveu "jogos" nos quais a maximização da pontuação da RAE prevalecia sobre outras considerações acadêmicas e as instituições tentavam aumentar sua pontuação contratando "estrelas" de seus rivais à maneira dos clubes de futebol. A RAE não só promoveu a competição entre instituições, mas deteriorou as relações entre colegas dentro das instituições quando indivíduos foram acusados de "decepcionar a equipe" e assediados por gerentes de linha carentes em habilidades interpessoais, apesar do treinamento que receberam nesta área, muitas vezes terceirizados para empresas privadas. Isso lançou, assim, as bases para um regime de "mecanismos de controle 
competitivos" na vida acadêmica, que continuou a apertar seu porão (Watermeyer 2019).

Dados os custos e os encargos administrativos de um sistema baseado na revisão por pares, mudanças ao longo do tempo incluíram o agrupamento de duas ou mais unidades de avaliação como subcomitês de um comitê principal. Isto teve alguma justificativa acadêmica em termos de reconhecimento da pesquisa interdisciplinar e de obrigar as disciplinas a conceber critérios em outros campos, mas para a próxima avaliação, a ser concluída em março de 2021, o número de subcomitês foi reduzido para 34, havendo apenas 4 painéis principais, enquanto que nos subcomitês RAE de 2008, 67 subcomitês trabalharam sob a orientação de 15 painéis principais. A crescente ênfase na excelência em nível internacional levou a que o financiamento disponível fosse concedido de forma cada vez mais seletiva, favorecendo as grandes universidades de pesquisa, enfraquecendo a capacidade do sistema de duplo apoio de dar a todos os acadêmicos alguma oportunidade de realizar pesquisa e ajudar os centros de excelência menores em instituições menos prestigiadas a se desenvolverem.

Após a RAE 2008, a organização que representa as instituições universitárias, Universities $U K$, pressionou para que as avaliações especializadas por pares das publicações fossem substituídas por uma avaliação menos custosa e administrativamente onerosa com base em dados bibliométricos. Mas foi decidido que a revisão por pares de especialistas deveria continuar sendo o principal método de avaliação para o renomeado Research Assessment Framework [Estrutura de Avaliação de Pesquisa] (REF) de 2014, embora os julgamentos também pudessem ser "balizados" por métricas em áreas temáticas para as quais isto fosse apropriado. Esta decisão foi tomada após extensas consultas, publicação de relatórios por órgãos como a Academia Britânica e a encomenda de estudos-piloto. O argumento de que a análise bibliométrica funcionava melhor para as ciências naturais e a matemática do que para as ciências sociais e humanas já estava consolidado. Em termos de cálculo de pontuação de citação e "fatores de impacto", acadêmicos que publicavam principalmente livros e capítulos de livros estavam em desvantagem em relação àqueles que publicavam principalmente em revistas. No entanto, o mesmo aconteceu com aqueles que mais divulgavam suas pesquisas através de anais de congressos, como os pesquisadores da computação. $\mathrm{O}$ fato que havia problemas em basear a avaliação em medidas 
bibliométricas mesmo dentro das ciências foi um dos assuntos debatidos em uma conferência internacional de especialistas em métrica em Leiden em 2014, cujas conclusões foram publicadas como o Manifesto de Leiden na revista Nature (Hicks, Wouters, et al. 2015).

Observando que algumas universidades, da Escandinávia à China, estavam agora alocando fundos de pesquisa ou pagando bônus com base em pontuações individuais de impacto ou publicação em "revistas de alto impacto", um critério cada vez mais utilizado também em casos de recrutamento e promoção, o Manifesto Leiden reconhece que as métricas podem ser úteis para reduzir o enviesamento na revisão pelos pares, mas fornece uma lista de razões pelas quais a tomada de decisões não deve ser "entregue aos números", mesmo que seja claro o que eles significam, o que está longe de ser o caso dadas as diferenças nas práticas de citação entre subcampos (mesmo nas ciências naturais e aplicadas) e os sérios vieses inerentes à construção de dados métricos. $\mathrm{O}$ primeiro grande banco de dados de citações, que se tornou a Web of Science, de propriedade da Thompson Reuters, era muito seletivo, muitas vezes em bases não muito transparentes, a respeito dos periódicos que escolhia indexar, mesmo dentro do mundo anglófono. Seu rival, o Scopus, de propriedade da Elsevier, apresentava problemas semelhantes. As taxas de citação e os fatores de impacto para os periódicos diferem muito entre os campos. As diferenças nas práticas de citação afetam as pontuações do índice h dos estudiosos individuais que trabalham em campos diferentes, mas são importantes também as diferenças na forma como as diversas bases de dados são construídas: pode haver grandes diferenças nas pontuações recebidas por indivíduos, por exemplo, na Web of Science e no relativamente mais inclusivo Google Scholar. ThompsonReuters tentou responder, através de sua parceria com a SciELO, às críticas da América Latina de que a produção acadêmica da região havia sido excluída de seu índice, mas mesmo essa parceria significou que apenas uma fração da pesquisa da região obteria um fator de impacto. Havia grandes esperanças que "altmetrics", métricas de nível para artigos capturadas de fontes como blogs, mídia social e Wikipedia, pudessem fornecer uma solução mais democrática globalmente. No entanto, ainda há problemas em saber o que significam essas medições (o que implica saber mais sobre os diferentes tipos de "públicos" que realmente leem os artigos), bem como os vieses 
do Atlântico Norte quanto à propriedade e uso de fontes altmétricas (Alperin 2014). Como o grupo de Leiden observou, o viés de publicação em língua inglesa nas revistas "de alto impacto" do Norte como critério de avaliação dos estudiosos em todo o mundo ignora a forma como uma boa parte da pesquisa no Sul está focalizada em tipos específicos de problemas regionais e nacionais e informando a política local, especialmente nas ciências sociais. Não apenas a excelência deve ser protegida na pesquisa "localmente relevante", mas desempenho precisa ser medido em relação às missões específicas de pesquisa de pesquisadores e instituições, já que fazer avançar as fronteiras do conhecimento acadêmico não é o mesmo que oferecer soluções para problemas sociais (Hicks, Wouters, et al. 2015:430).

O Brasil, como muitos outros países e a União Européia, tem seu próprio sistema de classificação de periódicos, mas as consequências perversas para além do viés do Atlântico Norte que podem surgir desses sistemas são consideráveis, e podem ocorrer mesmo em sistemas baseados em comitês de revisão por pares, já que os julgamentos dos revisores ainda podem ser influenciados por suas ideias sobre periódicos e editoras "líderes" em sua área. Um caso notório no Reino Unido é o da Economia. Os critérios do comitê levaram à extinção efetiva da "economia heterodoxa" sob a RAE, reforçando o domínio de um seleto grupo de departamentos que promovem a ortodoxia neoclássica (Lee, Pham \& Gu 2013). Os estudantes de Manchester fizeram manchetes nacionais ao exigir o ensino em abordagens heterodoxas, mas continuaram insatisfeitos com a resposta. Como as consequências econômicas da pandemia ameaçam aprofundar os problemas pré-existentes de desigualdade nas sociedades capitalistas, uma visão mais estratégica sugeriria que agora precisamos de todo o pensamento heterodoxo que pudermos obter neste campo.

Exercícios de análise comparativa internacional podem promover uma abordagem mais estratégica e deliberativa de avaliação. Em 2005, presidi o Comitê Diretor da primeira experiência da ESRC em análise comparativa internacional. Embora o chefe da ESRG à época, Ian Diamond, fosse um eminente estatístico social, ele foi tão insistente quanto eu que a avaliação deveria ser qualitativa. $\mathrm{O}$ trabalho do comitê internacional encarregado de realizá-la foi balizado por dados estatísticos sobre as particularidades da antropologia britânica e a colocação profissional de seus graduados compilados por 
David Mills, baseando-se no trabalho que a ASA já havia feito, mas a principal metodologia era baseada em visitas a um grupo diversificado de departamentos de antropologia. Isto permitiu que a equipe discutisse longamente com professores e estudantes e aplicasse suas habilidades etnográficas, assegurando aos colegas que este não era um exercício competitivo como a RAE. As visitas foram seguidas por sessões deliberativas que discutiam os resultados. Como o presidente do comitê internacional, Don Brenneis, já publicou um relato desta experiência no quadro de uma discussão comparativa mais ampla sobre a avaliação da antropologia (Brenneis 2009), vou me limitar a uma conclusão presente no relatório final (ESRC 2006). A antropologia britânica não só foi considerada líder mundial em diversas áreas acadêmicas, mas "extraordinária" nas interfaces que tinha construído entre o mundo acadêmico e áreas onde habilidades antropológicas e sensibilidades poderiam ser aplicadas para, comprovadamente, reformular a política, em particular, o desenvolvimento internacional. A ênfase dominante em publicações acadêmicas por parte da RAE foi um empecilho para que voltassem para a academia doutores em antropologia que haviam ido trabalhar em agências de desenvolvimento, mas os acadêmicos que trabalharam com agências de desenvolvimento não perderam sua capacidade de análise crítica construtiva sobre a forma como essas organizações trabalhavam. Infelizmente, o governo Johnson absorveu o Departamento de Desenvolvimento Internacional no interior do Ministério das Relações Exteriores, e os iminentes cortes no orçamento para subsídios provavelmente afetarão os programas de colaboração em pesquisa internacional por ele financiados e dos quais o Brasil tem sido um grande beneficiário.

A principal inovação na REF 2014 foi de fato a introdução do "impacto" não acadêmico como medida, contribuindo com vinte e cinco por cento para a nota final atribuída a cada unidade de avaliação, embora este tipo de "impacto" não tenha sido exigido para todas as pesquisas feitas na unidade. Os argumentos em favor do "impacto" foram feitos através de uma narrativa. Watermeyer (2019) argumenta que o que contava na avaliação de impacto era a elegância e plausibilidade das narrativas oferecidas, uma vez que a ausência de critérios claros desestimulava as tentativas de fazer uma avaliação rigorosa das reivindicações feitas nelas. Stein (2018) argumenta que o problema com a validação do impacto era que ele tinha que tomar a forma de reconhecimento escrito de uma 
instituição, governamental ou não-governamental, um procedimento inadequado para muitas formas de engajamento antropológico com as pessoas que estudamos. Ele também observa que a ênfase em projetos de pesquisa deixa de lado os impactos não acadêmicos do treinamento de estudantes que irão seguir carreiras fora da antropologia e do meio acadêmico. Mitchell (2014) aponta que havia suposições implícitas neste processo de auditoria sobre o que constituía "bom" impacto em termos de "efeito, mudança e benefício para a sociedade ou para a economia". Perguntou se os poderes dirigindo o sistema de controle considerariam o ativismo anticapitalista radical do falecido David Graeber como "bom impacto", mas também apontou para o problema não discutido de possíveis "maus" impactos, a começar pela relação de Napoleão Chagnon com os Yanomami. O que pode ser considerado "bom" ou "ruim" impacto depende claramente de um contexto de ideias predominantes. Tanto Mitchell como Stein mostram como e por que a avaliação do "impacto" não acadêmico permanece preocupante para uma consciência antropológica crítica, mesmo que isso seja o que muitos de nós almejam.

Para concluir. A forma como as universidades são "governadas" é um problema mais sério do que a métrica per se. Os números são essenciais em alguns tipos de pesquisa antropológica, especialmente se quisermos fazer uma contribuição ao debate de políticas públicas no mundo "pósverdade" de uma Internet que oferece todo tipo de alternativas perniciosas ao conhecimento fundamentado. No entanto, ao dividir-nos e fragmentar-nos através de uma cultura de auditoria de "controle competitivo" limita nossa capacidade de funcionar como intelectuais públicos e nega à sociedade o valor do que Tim Ingold define como conhecimento [scholarship], aquilo que une professores e estudantes em uma comunidade acadêmica dedicada a fazer novas perguntas e a buscar, coletivamente, novas respostas.

\section{Referências bibliográficas}

ALPERIN, Juan Pedro. 2014. "Altmetrics Could Enable Scholarship from Developing Countries to Receive Due Recognition." LSE Blogs, March 10, 2014, https://blogs.lse.ac.uk/impactofsocialsciences/2014/03/1 0/altmetrics-for-developing-regions/. 
BRENNEIS, Don. 2009. "Anthropology in and of the Academy: Assessment, and Our Field's Future". Social Anthropology 17(3):261-75.

ESRC. 2006. International Benchmarking Review of UK Social Anthropology. https://esrc.ukri.org/files/research/researchand-impact-evaluation/international-benchmarkingreview-of-uk-social-anthropology/.

HICKS, Diana; WOUTERS, Paul; WALTMAN, Ludo; RIJCKE, Sarah de; RAFOLS, Ismael. 2015. "Bibliometrics: The Leiden Manifesto for Research Metrics". Nature 520:429-31.

LEE, Frederic S; PHAM, Xuan; GU, Gyun. 2013. "The UK Research Assessment Exercise and the Narrowing of UK Economics". Cambridge Fournal of Economics 37(4):693-717.

LEMKE, Thomas. 2001. "The Birth of Bio-Politics: Michel Foucault's Lecture at the Collège de France on Neo-Liberal Governmentality". Economy and Society 30(2):190-207.

MITCHELL, Jon P. 2014. "Anthropologists Behaving Badly? Impact and the Politics of Evaluation in an Era of Accountability". Etnográfica 18(2):275-97.

ROSE, Nikolas. 1999. Powers of Freedom: Reframing Political Thought. Cambridge: Cambridge University Press.

SIVERTSEN, Gunnar. 2017. "Unique, but Still Best Practice? The Research Excellence Framework (REF) from an International Perspective". Palgrave Communications 3(1):1-6.

SHORE, Cris; WRIGHT, Susan. 1999. "Audit Culture and Anthropology: Neo-Liberalism in British Higher Education". The Fournal of the Royal Anthropological Institute 5(4):557-575.

SHORE, Cris; WRIGHT, Susan. 2015. "Audit Culture Revisited: Rankings, Ratings, and the Reassembling of Society". Current Anthropology 56(3):421-44.

STEIN, Felix. 2018. "Anthropology's 'Impact': A Comment on Audit and the Unmeasurable Nature of Critique". Fournal of the Royal Anthropological Institute 24(1):10-29.

STRATHERN, Marilyn. 1996. "From Improvement to Enhancement: An Anthropological Comment on the Audit Culture". The Cambridge Fournal of Anthropology 19(7):1-21. 
STRATHERN, Marilyn (ed.). 2000. Audit Cultures: Anthropological Studies in Accountability, Ethics and the Academy. London and New York: Routledge.

WATERMEYER, Richard. 2019. Competitive Accountability in Academic Life: The Struggle for Social Impact and Public Legitimacy. Cheltenham: Edward Elgar Publishing.

Enviado: 24 de novembro de 2020 Aceito: 1 de dezembro de 2020 